\title{
Apsidal motion in the massive binary HD 152248
}

\author{
S. Rosu ${ }^{1}$, G. Rauw ${ }^{1}$, E. Gosset ${ }^{1}$, J. Manfroid ${ }^{1}$ and P. Royer ${ }^{2}$ \\ ${ }^{1}$ Space sciences, Technologies and Astrophysics Research (STAR) Institute, \\ Université de Liège, Allée du 6 août 19c, Bât B5c, 4000 Liège, Belgium, \\ (E-mail:sophie.rosu@uliege.be) \\ 2 Instituut voor Sterrenkunde, KU Leuven, Celestijnenlaan 200D, Bus 2401, \\ 3001 Leuven, Belgium
}

Received: September 29, 2019; Accepted: December 17, 2019

\begin{abstract}
The eccentric massive binary HD 152 248, hosting two very similar O7.5 III(f) stars, is the most emblematic eclipsing O-star binary in the very young and rich open cluster NGC 6231. Measuring the rate of apsidal motion in such a binary system gives insight into the internal structure and evolutionary state of the stars composing it.

Key words: stars: early-type - stars: individual (HD 152 248) - stars: massive

- binaries: spectroscopic - binaries: eclipsing
\end{abstract}

\section{Introduction}

The majority of massive stars belong to binary systems. In double-line spectroscopic eclipsing binaries, combining the photometric eclipses and the radial velocities obtained with spectroscopy allows to determine the masses and radii of the stars in a model-independent way. If the binary is eccentric and shows a significant apsidal motion, measuring this rate provides a diagnostic of the internal mass-distribution of the stars, which is otherwise difficult to constrain. It also offers a test of our understanding of stellar structure and evolution.

\section{Methods and results}

From a set of optical spectra of HD 152248 , we reconstruct the individual spectra of the stars and establish their radial velocities using a disentangling code. We analyse the reconstructed spectra with the CMFGEN model atmosphere code (Hillier \& Miller, 1998) to determine stellar and wind properties of the system, notably effective temperatures $\mathrm{T}_{\text {eff }}$ of $34000 \pm 1000 \mathrm{~K}$ and $\log g=$ $3.48 \pm 0.10$ (cgs) for both stars. We stress that the disentangling method introduces artefacts in the wings of broad lines. In addition, CMFGEN does not account for binarity. Hence, $\log g$ is underestimated and $\mathrm{T}_{\text {eff }}$ is only an average over the visible surface of the stars. The optical light curve of the binary is analysed with the Nightfall binary star code (Wichmann, 2011) to constrain the Roche lobe filling factors of both stars to a value of 0.86 and derive an orbital 
inclination of $\left(68.6_{-0.3}^{+0.2}\right)^{\circ}$. Nightfall uses the Roche potential to describe the shape of the stars, accounts for reflection effects (mutual irradiation) and adopts a quadratic limb-darkening law. Absolute masses of $28.9_{-0.8}^{+0.9}$ and $29.1_{-0.5}^{+0.9} \mathrm{M}_{\odot}$ are derived for the primary and secondary star respectively and mean stellar radii of $14.2 \pm 0.4 R_{\odot}$ are obtained for both stars. Combining radial velocity measurements spanning seven decades (see Fig. 1), we show that the system displays an apsidal motion at a rate of $\left(1.750_{-0.315}^{+0.350}\right)^{\circ} \mathrm{yr}^{-1}$.
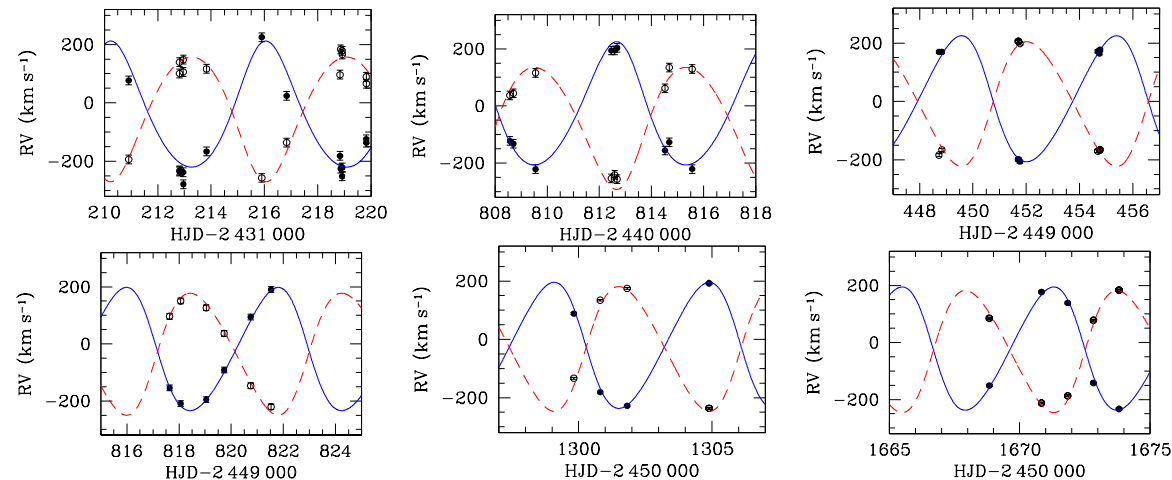

Figure 1. Comparison between the measured RVs of the primary (filled dots) and secondary (open dots) and the RV curves obtained with the best-fit parameters. Top panels correspond, from left to right, to data from Struve (1944), Hill et al. (1974) and one epoch of data from Mayer et al. (2008). Bottom left panel yields one epoch of $I U E$ data from Penny et al. (1999) while bottom middle and right panels correspond to RVs re-derived in this work (see Rosu et al. 2020, accepted).

\section{Conclusion}

The properties of HD 152248 render the system an interesting target to study tidally induced apsidal motion from a theoretical point of view. As a next step, we will build stellar evolution models to determine a theoretical rate of apsidal motion, which could, by comparison with the observed rate, allow to constrain the internal mixing processes occurring inside the stars as well as to infer an age estimate for the binary system.

Acknowledgements. S. Rosu is supported by the FNRS (Fonds National de la Recherche Scientifique) - FNRS PhD grant.

\section{References}

Hill, G., Crawford, D.L., \& Barnes, J.V. 1974, Astron. J., 79, 1271

Hillier, D.J., \& Miller, D.L. 1998, Astrophys. J., 496, 407 
Mayer, P., Harmanec, P., Nesslinger, S., et al. 2008, Astron. Astrophys., 481, 183

Penny, L.R., Gies, D.R., \& Bagnuolo, W.G.Jr. 1999, Astrophys. J., 518, 450

Rosu, S., Rauw, G., Conroy, K.E., Gosset, E., Manfroid, J., \& Royer, P. 2020, Astron. Astrophys., 635, A145, DOI: 10.1051/0004-6361/201937285

Struve, O. 1944, Astrophys. J., 100, 189

Wichmann, R. 2011, Nightfall: Animated Views of Eclipsing Binary Stars, Astrophysics Source Code Library, record ascii:1106.016 